\title{
Médiévales
}

Langues, Textes, Histoire

78 | printemps 2020

Moyen Âge en séries

\section{Médiévalisme boréal et séries télévisées}

\section{Pierre-Brice Stahl}

\section{OpenEdition \\ Journals}

Édition électronique

URL : https://journals.openedition.org/medievales/10777

DOI : 10.4000/medievales. 10777

ISSN : 1777-5892

\section{Éditeur}

Presses universitaires de Vincennes

\section{Édition imprimée}

Date de publication : 20 août 2020

Pagination : $57-68$

ISBN : 978-2-37924-093-5

ISSN : 0751-2708

\section{Référence électronique}

Pierre-Brice Stahl, « Médiévalisme boréal et séries télévisées », Médiévales [En ligne], 78 | printemps 2020, mis en ligne le 02 janvier 2022, consulté le 22 avril 2022. URL : http://journals.openedition.org/ medievales/10777; DOI : https://doi.org/10.4000/medievales.10777 


\section{Pierre-Brice Stahl}

\section{Médiévalisme boréal et séries télévisées}

Les Vikings et les divinités nordiques connaissent une grande popularité ces dernières années, avec, entre autres, une présence significative dans les industries culturelles et créatives. Le présent article s'intéresse à cet emploi du Nord médiéval dans les séries télévisées exogènes, à savoir les œuvres qui sont produites en dehors de la sphère nordique ${ }^{1}$. En effet, les imaginaires géographiques et historiques de ces pays sont différents dans une perspective endogène, où ils représentent à la fois une réalité observable et un héritage culturel identitaire lié à la mémoire ${ }^{2}$.

La notion de Nord médiéval renvoie communément à la période viking dont les bornes chronologiques traditionnelles vont de 793 à $1066^{3}$. L'imaginaire qui lui est associé est à la jonction de plusieurs discours dans lesquels il s'inscrit et auxquels il participe : discours sur la période médiévale, sur le Nord, sur les mythes nordiques, sur les Vikings, etc. Nous retrouvons ainsi trois autres imaginaires qui interagissent: un temporel

1. L'ensemble des séries étudiées répond aux quatre critères du présent dossier : sérialité, production pour les écrans, dimension fictionnelle et rapport au Moyen Âge. Les dessins animés, qui présentent d'autres codes et modes de production, ne seront pas traités dans cette étude.

2. Sur le concept d'endogène et d'exogène, voir A. BALLOTTI, «Analyse des processus d'interaction et de réception du boréalisme ", dans S. BRIENS et P.-B. STAHL éd., Boréalisme 2.0, Études Germaniques, 290 (2018), p. 177-192. Parmi les séries nordiques, nous pouvons citer, à titre d'exemple, Hem til Midgård (2003-2004) ; Jul i Valhal (2005) ; Arn (2010); Vikingane (2016).

3. Le Nord médiéval n'est toutefois pas strictement lié à cette période. Il renvoie également aux divinités nordiques ou à des figures historiques qui peuvent se détacher de celle-ci. 
(le médiévalisme), un géographique (le boréalisme ${ }^{4}$ ) et un ethnique (lié à l'idée d'un peuple). Le médiévalisme boréal s'intéresse au processus de fabrication et à la réception de l'imaginaire du Nord médiéval. Ce dernier se caractérise par sa plasticité et son hétérogénéité qui se construit également par l'interaction des visions exogènes, endogènes et académiques du Nord. C'est à travers ce cadre conceptuel que je propose de présenter et d'analyser l'emploi du Nord médiéval dans les séries télévisées ${ }^{5}$.

\section{Histoire et production des séries}

Si les premières séries qui font appel au Nord médiéval apparaissent dès la fin des années 1950, elles sont pourtant relativement rares jusqu'aux abords de l'an 2000. Une série entière lui est toutefois consacrée entre 1959 et 1960 : Tales of the Vikings ${ }^{6}$, où l'on suit les aventures de Leif Ericson. Nous retrouvons également les Vikings dans The Adventures of Sir Lancelot (1956-1957), avec l'épisode 17, «The Lesser Breed», ainsi que dans le neuvième feuilleton de la saison 2 de Doctor Who (1963-1989), « The Time Meddler ${ }^{7} »$, diffusé en quatre épisodes ${ }^{8}$. Cette première phase est suivie d'une longue période où le Nord médiéval ne semble plus être employé comme motif dans les intrigues ${ }^{9}$. Mis à part le quatrième feuilleton de la saison 26 de Doctor Who, «The Curse of Fenric ${ }^{10}$ », il faut attendre 1997 avec Stargate SG-1 (1997-2007), puis Hercules : The Legendary Journeys $(1995-1999)^{11}$ et son spin off Xena: Warrior Princess (1995-2001),

4. Le concept de boréalisme, développé en France par Sylvain Briens, peut se définir comme le processus de fabrication du Nord. Voir S. BRIENS et P.-B. STAHL éd., Boréalisme 2.0,... ; S. BRIENS éd., Le Boréalisme, Paris, 2016 (Études germaniques, 71).

5. Ce concept s'inscrit dans le cadre du projet Boréalisme de l'EA 3556 REIGENN dirigé par S. BRIENS et fera l'objet d'une monographie, intitulée Le Médiévalisme boréal, par l'auteur en 2021.

6. Il a été choisi de garder le titre d'origine des séries citées.

7. Doctor Who, BBC Studios, 1963-1989, « The Time Meddler » (feuilleton 9) : « The Watcher », 3 juillet 1965, «The Meddling Monk», 10 juillet 1965, «A Battle of Wits», 17 juillet 1965, et «Checkmate », 24 juillet 1965. Doctor Who se divise en deux séries, la première de 1963 à 1989 et la seconde diffusée depuis 2005 (en production).

8. Il est également possible de noter « The Ceremony of Innocence » qui, en termes de format, correspond à un film télévisé, mais fait partie de la série NET Playhouse (S4/E24, 12 juin 1970).

9. Les phases introduites dans cet article prennent pour perspective l'histoire générale de l'utilisation du Nord médiéval dans les séries exogènes. Ces phases sont donc à recontextualiser pour chaque pays en prenant en compte les éventuelles dates de diffusions.

10. Le feuilleton est divisé en quatre parties sans titres spécifiques. Il a été diffusé entre le 25 octobre 1989 et le 15 novembre 1989.

11. Hercules: The Legendary Journeys, Renaissance Pictures, 1995-1999, S5/E6, «Norse By Norsevest» (2 novembre 1998); S5/E7, «Somewhere Over the Rainbow Bridge $»(9$ novembre 1998). 
pour le voir réapparaître ${ }^{12}$. Cette troisième phase est caractérisée par une augmentation significative en l'espace de quelques années de l'emploi du Nord médiéval, qui se retrouve au sein d'arcs narratifs dans de nombreuses séries. Ainsi l'épisode «Thor's Hammer » de Stargate $S G$ - 1 introduit les Asgards, une race d'extra-terrestres qui seront présents ou mentionnés dans plusieurs épisodes de la série et dans son spin off Stargate Atlantis (2004-2009). Comme nous allons le voir, cette troisième phase témoigne d'emplois très variés du Nord médiéval et comprend notamment les séries Dark Ages (1999), There's a Viking in My Bed (2001), Entourage (2004), Supernatural (2005-...), True Blood (2008-2014), The Vampire Diaries (2009-2017). Les années 2010 marquent la quatrième phase qui, tout en prolongeant la précédente, se caractérise par l'emploi du Nord médiéval au centre de l'intrigue ${ }^{13}$. Entre 2011 et 2018, six séries en font un élément constitutif de l'intrigue principale : The Almighty Johnsons (2011), Vikings (2013-2020), The Last Kingdom (2015-...), American Gods (2017-...), Victor Magtanggol (2018), Galères Vikings (2018).

L'histoire de ces séries est également liée à celle d'autres médias. Elle s'insère donc dans une réception plus large des productions artistiques $\mathrm{du} \mathrm{XX}^{\mathrm{e}}$ et $\mathrm{du} \mathrm{XXI}^{\mathrm{e}}$ siècle. Ainsi, la série Tales of the Vikings a été produite par la compagnie de Kirk Douglas, Bryna Productions, suite au succès du film de 1958, The Vikings, qui se fonde sur le roman d'Edison Marshall de 1951. Plusieurs séries employant le Nord médiéval sont également des adaptations de romans : There's a Viking in My Bed reprend l'ouvrage pour enfants du même nom de Jeremy Strong ${ }^{14}$; Witches of East End adapte le roman éponyme de Melissa de la $\mathrm{Cruz}^{15}$; The Last Kingdom se fonde sur The Saxon Stories de Bernard Cornwell ${ }^{16}$; American Gods est issue du roman de Neil Gaiman ${ }^{17}$. Cette pratique de la crossmédialité, qui nécessite d'être prise en compte pour toute analyse, n'est bien entendu pas une spécificité des séries sur le Nord médiéval ${ }^{18}$. Les productions artistiques n'adaptent

12. Xena : Warrior Princess, S6/E7, «The Rheingold» (13 novembre 2000); S6/E8, « The Ring » (20 novembre 2000); S6/E9, « Return of the Valkyrie » (27 novembre 2000); S6/E13, « You Are There » (5 février 2001).

13. Cette phase actuelle poursuit les emplois de la précédente avec notamment les séries Supernatural, Doctor Who, The Vampire Diaries et son spin off The Originals (2013-2018).

14. J. STRONG, There's a Viking in My Bed, Londres, 1990.

15. Il s'agit du premier roman de la série The Beauchamp Family qui donne une plus grande place au Nord médiéval que son adaptation télévisuelle: M. DE LA CRUZ, The Beauchamp Family (série), vol. 1 : Witches of East End, New York, 2011.

16. B. CORNWELL, The Saxon Stories (série), 12 volumes publiés, Londres, 2004-en cours.

17. N. Gaiman, American Gods, New York, 2001.

18. Sur la crossmédialité et la transmédialité, voir M. LETOURNEUX, Fictions à la chaîne . Littératures sérielles et culture médiatique, Paris, 2017, et H. JENKINS, Convergence Culture. Where Old and New Media Collide, New York, 2006. 
pas uniquement les œuvres, mais les prolongent sur d'autres formats ou dans d'autres médias. Certaines séries que nous avons présentées sont ainsi de véritables œuvres transmédiatiques employant différents dispositifs comme les comics, les romans, les jeux vidéo, les dessins animés et les films pour développer leurs univers (on peut citer à titre d'exemple Stargate, Supernatural ou Doctor Who). Le Nord médiéval des séries télévisées va ainsi pouvoir se retrouver dans ces autres supports, comme l'illustre bien Kaamelott dont la première bande dessinée, Les Sièges de transport, place les Vikings au centre de l'intrigue et permet de prolonger le médiévalisme boréal de l'œuvre d'Alexandre Astier ${ }^{19}$.

Le Nord médiéval de ces séries se rattache à un large éventail de périodes et de lieux, souvent déterminés par les centres de production. En termes d'époques, il peut être placé dans un temps légendaire (Hercules: The Legendary Journeys, Xena : Warrior Princess, The Adventures of Sir Lancelot), à l'époque viking (Tales of the Vikings, True Blood, Vikings, The Last Kingdom), au présent (American Gods, There's a Viking in My Bed, Galères Vikings) ou dans le futur (univers Stargate). En termes d'espace, l'action peut se situer dans le Nord scandinave, mais également dans les lieux d'activités historiques des Vikings comme la Grande-Bretagne et l'Amérique du Nord, dans des lieux imaginaires, et enfin dans des lieux en dehors de toute activité viking, tels que les Philippines ou la Nouvelle-Zélande. Il y a bien entendu une corrélation entre le genre des séries, l'époque et le lieu de l'action ${ }^{20}$, mais également entre les lieux de productions et l'époque/ les lieux de l'action ${ }^{21}$. Ainsi l'action des séries situant le Nord médiéval en dehors des lieux traditionnels de l'activité viking se déroule dans les pays où elles sont produites : Galères Vikings (France, plus spécifiquement Strasbourg), Victor Magtanggol (Philippines), American Gods (États-Unis) et The Almighty Johnsons (Nouvelle-Zélande). Toutes ces productions ont la particularité de faire intervenir le Nord médiéval dans leurs époques (début du XXI ${ }^{\text {e }}$ siècle). Nous retrouvons également cette corrélation pour les zones d'activités des Vikings. Tales of the Vikings, série canadienne, s'intéresse à la figure de Leif Erikson, considéré comme le premier Européen à avoir découvert l'Amérique du Nord. Même si, à travers les figures de Ragnar Lothbrok et de ses fils, la série canado-irlandaise Vikings souhaite présenter l'ensemble de l'épopée viking et son expansion, l'exploration de l'Amérique du Nord était dès l'origine une des intentions majeures de

19. A. AstiER et S. DuPRÉ, Les Sièges de transport, Paris, 2007.

20. Voir par exemple les séries de science-fiction telles que Stargate.

21. C'est ce qui explique la rareté des séries situant l'action en Scandinavie médiévale, puisque le corpus de cet article ne prend pas en compte les séries endogènes. Nous pouvons toutefois évoquer le site fictionnel de Kattegat qui sert de point d'ancrage à la série Vikings. Voir P.-B.STAHL, « La représentation du Nord dans Vikings. Entre exploration, imaginaire et altérité », dans S. BRIENS et ID. éd., Boréalisme 2.0, p. 285-294 (p. 289). 
son créateur Michael Hirst ${ }^{22}$. Quant à The Last Kingdom, série britannique, elle présente le rôle de l'activité viking dans l'unification des royaumes anglo-saxons. Ces séries situent toutes l'action à l'époque viking. Le Nord médiéval sert d'identité narrative et participe à la mémoire culturelle des pays de production.

\section{L'hétérogénéité du Nord médiéval dans les séries}

Ces séries présentent différents types d'emploi du Nord médiéval. Il peut, par exemple, être au centre de l'intrigue et devenir un élément indissociable de la série, ou jouer un rôle sur un épisode, voire au sein d'un arc narratif plus long. Il est possible de distinguer d'autres utilisations, dont une que je propose d'assimiler à la touche en peinture. La touche peut prendre des natures et des aspects différents en fonction de l'outil employé, du support de l'œuvre, du mouvement dans lequel elle s'insère et de la manière de l'appliquer. Elle peut donc être invisible, discrète, imposante, etc. Cette utilisation du Nord médiéval n'affecte pas la narration de l'épisode ou de la série et peut être entièrement anodine. Il peut, par exemple, s'agir de la mention du marteau de Thor comme artefact dans «... And the Drowned Book » de The Librarians (2014-2018) ${ }^{23}$, d'une blague sur une mascotte et les Suédois dans Modern Family (2009-...) ${ }^{24}$, ou du port d'un costume de Viking par le Doctor dans l'épisode «Robot» de Doctor Who ${ }^{25}$. Ce type d'utilisation se retrouve ainsi dans une multitude de séries de genres différents.

En raison de son lien étroit avec les origines et le développement de la fantasy, des aspects du Nord médiéval se retrouvent également dans les séries qui font appel à ce genre. Ces références n'y sont pas forcément directement visibles ou même conscientes et peuvent nécessiter un travail d'archéologie des motifs, afin de pouvoir les identifier ${ }^{26}$. Généralement plus facilement décelables, les Vikings peuvent également être, dans ces séries, une source d'inspiration pour des peuples issus des mondes imaginaires de la fantasy. C'est notamment le cas des Ironborn (Fer-nés dans la version française) dans Game of Thrones (2011-2019), dont la société et la religion s'inspirent des peuples nordiques médiévaux.

Le Nord médiéval s'attache ainsi à des genres très différents. Si les premières manifestations s'insèrent dans des séries d'aventures, elles se

22. J. POllard, The World of Vikings, Avant-propos de M. HIRST, San Francisco, 2015, p. 160 .

23. S2/E1. La série mentionne également l'œil de Wotan (S3/E5) parmi plusieurs autres artefacts issus de différentes mythologies et légendes.

24. S10/E8.

25. S12, feuilleton 1 en quatre parties.

26. Le terme « archéologie » est ici employé avec le sens donné par Michel Foucault. 
retrouvent rapidement dans d'autres catégories, notamment en raison des tendances du marché. En dehors des séries dans lesquelles le Nord médiéval est une touche, on le retrouve dans des genres aussi variés que la comédie, la fantasy, le drame historique, l'horreur, l'aventure ou le surnaturel. Chacun de ces genres renvoie à des codes, des structures et des motifs spécifiques qu'il faut prendre en compte et qui affectent la manière d'utiliser le Nord médiéval. En plus des genres de l'œuvre, son emploi est en lien avec de multiples facteurs comme la période et le lieu de production, le contexte socio-économique, les techniques, etc. Ainsi le logo de la «Yggdrasill Corporation» (ユグドラシルコーポレーション) de la série Kamen Rider Gaim (仮面ライダー鎧武／ガイム), 2013-2014) renvoie à une esthétique bien spécifique du tokusatsu de super-héros ${ }^{27}$.

Le budget des séries a également des conséquences sur l'adaptation du Nord médiéval. La série Kaamelott choisit, par exemple, de narrer la flotte viking dans l'épisode 49 du livre IV intitulé «Drakkars ! » plutôt que de la représenter. Enfin, il est essentiel de prendre en considération les différentes étapes de production de ces séries qui font fréquemment appel à des compagnies extérieures pour le générique, l'affiche ou les effets spéciaux ${ }^{28}$. Le résultat final est ainsi issu d'une multitude d'acteurs décisionnels différents, qui peuvent représenter des intentions et des réceptions différentes.

La multiplicité de ces réceptions induit une grande variété du médiévalisme boréal. La figure du Viking permet de rendre compte de cette pluralité. On peut remarquer en premier lieu que le terme «viking », quand il est employé, ne renvoie pas à la même réalité en fonction des séries. Héritière d'un discours qui se crée au XIX ${ }^{\mathrm{e}}$ siècle, la majorité des productions emploie ce nom pour désigner l'ensemble des Scandinaves de l'époque médiévale. Certaines séries réservent toutefois le terme à la désignation de celui qui participe à une expédition maritime, conformément au sens du terme víkingr dans les sources norroises ${ }^{29}$, ou emploient un autre dénominatif. C'est le cas notamment de The Last Kingdom qui identifie l'envahisseur comme étant danois.

Le port ou non du casque à cornes induit également des visions différentes de la figure du Viking. Cette association, qui remonte notamment au XIX ${ }^{\mathrm{e}}$ siècle, s'est consolidée jusqu'à devenir un symbole évoquant à lui seul la figure du Viking ${ }^{30}$. C'est ainsi que les drames historiques tels que

27. Genre caractérisé par l'utilisation d'effets spéciaux.

28. À titre d'exemple, les séquences d'ouverture de Vikings et de The Last Kingdom ont été réalisées, respectivement, par les sociétés The Mill et Huge Designs.

29. D'où l'emploi possible du terme « viking » sans majuscule.

30. Sur la question du casque à corne et l'archéologie de ce motif, voir P.-B. STAHL, «"Dis donc toi, avec ton casque à cornes !" Le Viking et son casque », dans F. BESSON et J. Breton éd., Kaamelott, un livre d'histoire, Paris, 2018, p. 221-228 ; R. FrANK, « The 
Vikings et The Last Kingdom choisissent de ne pas employer cet attribut afin de jouer sur la notion d'authenticité historique de la série, qui peut devenir un argument marketing ${ }^{31}$. Le casque à cornes continue toutefois à être utilisé, notamment dans les comédies. On le retrouve ainsi dans Dark Ages, There's a Viking in My Bed, Entourage (avec la série fictionnelle Viking Quest) et Kaamelott. Il s'agit également d'un des emplois majoritaires dans les séries où le Viking apparaît en tant que touche. Ainsi Blackadder (1983-1989) fait des casques à cornes la raison d'une des «pires batailles de l'histoire »: les cornes avaient été installées par erreur à l'intérieur des casques ${ }^{32}$. Dans ces séries, la coiffe viking sert d'élément directement identifiable. Si le casque peut répondre à une association attendue du public, il s'est également détaché de la figure du Viking historique pour prendre une indépendance que l'on retrouve, par exemple, dans la fantasy ${ }^{33}$.

Le casque à corne est également un marqueur d'altérité. Celui-ci n'a d'ailleurs pas disparu, mais a évolué : du casque on est passé à la coiffure viking, popularisée notamment par Vikings. Pour les personnages masculins, elle consiste principalement en une coupe undercut qui peut être combinée avec un man bun (chignon d'homme), un top knot (nœud haut), des motifs tressés et une barbe. Une esthétique spécifique est également mise en place pour les coupes des personnages féminins, caractérisée, entre autres, par des tresses en diagonale ou en arrière, un assemblage de mèches en-dessous ou au-dessus de la tresse, des motifs torsadés, et une coupe sidecut. Cette altérité est également renforcée par l'emploi des tatouages sur le crâne et le reste du corps, ainsi que par les costumes. Par le travail des costumiers, des coiffeurs stylistes et des accessoiristes, toute une esthétique spécifique est mise en place qui se fonde notamment sur l'avancée de la recherche et la reconstitution. Ce lien avec le domaine académique et la reconstitution est un des aspects du médiévalisme boréal. Il permet, entre autres, de se démarquer des visions antérieures du Nord médiéval et d'apporter un gage d'authenticité à la série, ce qui pourra être utilisé pour sa promotion.

D'autres formes d'altérité sont également mises en avant à travers la société et la culture comme : l'opposition entre la religion préchrétienne et

Invention of the Viking Horned Helmet », dans M. DALlAPIAZZA et al. éd., International Scandinavian and Medieval Studies in Memory of Gerd Wolfgang Weber, Trieste, 2000, p. 199-208.

31. Voir, par exemple, la série documentaire The Real Vikings (2016), produite par la chaîne History, où des acteurs de la série rencontrent des experts académiques, ou encore l'ouvrage The World of Vikings (voir n. 22).

32. S4/E3 (12 octobre 1989) : «A war hasn't been fought this badly since Olaf the Hairy, High Chief of all the Vikings, accidentally ordered 80,000 battle helmets with the horns on the inside. » Nous retrouvons également dans cette citation la vision d'un peuple viking homogène sous la régence d'un roi unique.

33. P.-B. STAHL, «"Dis donc toi, avec ton casque à cornes !"... », p. 227-228. 
le christianisme ${ }^{34}$; une certaine indépendance des femmes qui va s'opposer à l'imaginaire traditionnel de leurs rôles à l'époque médiévale ${ }^{35}$; les paysages. Dans le cadre des séries où les Scandinaves interagissent avec d'autres peuples médiévaux, une double altérité se met ainsi en place pour le spectateur.

\section{L'imaginaire géographique du Nord}

Le médiévalisme boréal offre au téléspectateur une identité visuelle directement reconnaissable, en opposition avec les représentations sur le Moyen Âge continental. Ainsi, à l'instar du Proche-Orient médiéval, nous allons trouver une esthétique qui va se poser en véritable altérité et offrir un contraste avec l'espace médiéval anglo-saxon et continental. Si l'imaginaire de ce dernier fait appel à des motifs tels que la forêt, l'agriculture, la cathédrale, le village ou la ville fortifiée, et le château, le médiévalisme boréal recourt quant à lui au fjord, à la brume, à la neige, à la maison longue et à la mer. En dehors de ces éléments, les séries emploient également des techniques cinématographiques pour traduire cette altérité ou pour marquer la spécificité du Nord médiéval. La série Vikings, par exemple, utilise des teintes, des saturations et des luminosités différentes selon que l'action se déroule en Scandinavie, dans les royaumes anglo-saxons ou en Francie occidentale ${ }^{36}$. Demi-dioptrie, filtres, lentilles anamorphiques, etc., le directeur de la photographie dispose d'un vaste choix d'outils et de techniques pour mettre en place une atmosphère spécifique liée au Nord médiéval. Aux paysages visuels viennent également s'ajouter les paysages sonores. La conception sonore participe à cette atmosphère du Nord et comprend aussi bien les bruits de l'environnement que les créations musicales.

Le Nord représenté dans les séries emploie tout un réseau d'images qui véhiculent, entre autres, l'idée d'un Nord homogène. En effet, si les représentations se fondent en partie sur des réalités géographiques et climatiques, elles vont être appliquées à l'ensemble du Nord médiéval, créant un topos imaginaire unique, lieu de résidence des Vikings. Ce territoire prend, par exemple, le nom de Norseland dans Hercules: The Legendary Journeys, Xena: Warrior Princess et Dark Ages. La série Vikings, quant à elle, débute avec pour seule indication géographique le terme «Scandinavia ${ }^{37}$ ». L'emploi d'un terme unique pour désigner l'ensemble des régions scandinaves accentue cette notion d'homogénéité et

34. Voir The Last Kingdom et Vikings. [Voir aussi l'article de R. FACCHINI et D. IACONO dans le présent numéro (NDLR).]

35. Voir P.-B. STAHL, « La représentation du Nord... », p. 293-294.

36. Ibid., p. 292-293.

37. S1/E1, « Rites of Passage». 
sert de lieu d'évocation sur lequel est projetée l'idée d'un Nord médiéval. Ces espaces imaginaires spécifiques convoquent tout un ensemble de motifs et se détachent des réalités géographiques des lieux ${ }^{38}$. Les séries puisent dans cette réserve d'éléments qui comprend, entre autres, la mer, le fjord, la brume, la tempête, les montagnes et la neige ${ }^{39}$. Le personnage d'Ashildr interprété par Maisie Williams dans Doctor Who (2005-...) reprend bien cette vision dans la description de son environnement : « This is my place, the sky, the hills, the sea, the people ${ }^{40}$. » De plus, les raisons pratiques et économiques liées aux tournages des séries font que les scènes sont rarement tournées dans le Nord ${ }^{41}$.

Les génériques participent également à cet imaginaire du Nord et jouent un rôle important : présents dans l'ensemble des épisodes, ils viennent à symboliser la série dont ils peuvent refléter l'identité et le genre. Ils sont généralement issus de studios de production spécialisés qui cherchent à transmettre l'essence de la série durant les quelques secondes de la séquence d'ouverture. Les quatre séries télévisées où la figure du Viking est au centre de la narration font intervenir un navire viking dans le générique ${ }^{42}$. La série Tales of the Vikings s'ouvre et se termine à chaque épisode par une séquence présentant un navire voguant à la voile dans un fjord ${ }^{43}$. Il est intéressant de noter que, même si l'action se déroule essentiellement dans le village côtier de Flotby et dans son auberge, la séquence d'ouverture de There's a Viking in My Bed présente un navire dans une mer déchaînée ${ }^{44}$. Les génériques de Vikings et The Last Kingdom s'ouvrent également sur des navires vikings pris dans une mer mouvementée ${ }^{45}$. Ce motif du navire dans la tempête est un des éléments principaux attachés à la figure du Viking ${ }^{46}$. Il transmet une

38. Il est également possible de parler de mythèmes au sens proposé par Thomas Mohnike qui distingue entre des mythèmes acteurs, d'actions, de cadre, et de concept : T. MoHNIKE, « Narrating the North. Towards a Theory of Mythemes of Social Knowledge in Cultural Circulation », à paraître. Voir le programme de recherche « Mythèmes du Nord » [http://mythemes.u-strasbg.fr/].

39. D. Chartier, «Qu'est-ce que l'imaginaire du Nord? », dans S. BrIENS éd., Le Boréalisme, p. 189-200 (p. 189-190).

40. S9/E5, « The Girl Who Died».

41. Voir par exemple l'utilisation du matte painting digital (travail de l'image à partir de prises de vues réelles) pour la série Vikings : P.-B. STAHL, « La représentation du Nord... », p. 190-191.

42. À savoir Tales of the Vikings, There's a Viking in My Bed, Vikings et The Last Kingdom. On retrouve également le navire viking dans le générique de Victor Magtanggol.

43. Cette esthétique est renforcée par les paroles du thème principal de la série, partie intégrante du générique. Voir les premiers vers : «From out of the North like an icy wind / Came Odin's fair warriors breed ».

44. Ce navire permet également d'expliquer l'arrivée du Viking à Flotby.

45. Pour une analyse du générique de Vikings, voir P.-B. STAHL, « La représentation du Nord... », p. 187.

46. Voir par ex. J.-M. LÉVESQuE éd., Dragons et Drakkars. Le mythe viking de la Scandinavie à la Normandie, XVIII $-X X^{e}$ siècles, Caen, 1996. 
atmosphère dramatique, épique et devient un véritable élément narratif à lui seul.

Cet imaginaire géographique du Nord médiéval transmis par les séries se rattache à des discours endogènes et exogènes anciens ainsi qu'à leurs interactions. Ce processus dynamique de fabrication de l'imaginaire du Nord, le boréalisme, donne à observer l'idée du Nord que fabriquent et projettent à leurs tours les séries. Ainsi, le lien entre le Viking et les forces de la nature se place notamment dans la continuité discursive du romantisme national suédois. Dans son poème Vikingen, Gustaf Geijer (1783-1847) recourt, par exemple, à ce motif de la mer déchaînée : «Ainsi, jeté par un naufrage sur l'écueil isolé, le Viking chantait au sein des flots orageux ${ }^{47}$. » Ces vers s'appliquent parfaitement à l'épisode 17, « The Lesser Breed », de la série The Adventures of Sir Lancelot, où les vagues s'abattent sur le personnage d'Eck qui reste impassible. À travers leurs emplois de motifs tels que la brume, la mer déchaînée, la nature sauvage, les séries se placent dans une continuité discursive boréaliste ${ }^{48}$. Elles prolongent des modèles esthétiques hérités, entre autres, du sublime, du gothicisme et du romantisme qui apportent une des spécificités du médiévalisme boréal et participent à son altérité.

Par leur emploi de l'imaginaire du Nord médiéval qu'elles resémantisent, les séries télévisées participent à un discours médiévaliste boréal qui se place en altérité avec l'imaginaire traditionnel de l'Europe médiévale. Le médiévalisme boréal de ces séries se construit à travers l'interaction d'imaginaires (temporels, géographiques et ethnique), de discours (endogènes, exogènes et académiques) et de visions différentes. C'est ainsi un Nord médiéval hétérogène qui se présente au téléspectateur, en lien notamment avec les genres et les contextes de productions de chaque série.

Pierre-Brice Stahl - Sorbonne Université

47. Vikingen d'Erik Gustaf Geijer, trad. par X. MARMIER, Chants populaires du Nord, Paris, 1842, p. 291.

48. Ces motifs correspondent aux mythèmes de cadres d'après la nomenclature proposée par le projet Mythèmes du Nord (voir n. 38). 


\section{Médiévalisme boréal et séries télévisées}

À partir du concept de médiévalisme boréal, le présent article propose une analyse de l'emploi du Nord médiéval dans les séries télévisées produites en dehors de la sphère nordique. Ces dernières témoignent de l'hétérogénéité et de la plasticité de l'imaginaire sur le Nord médiéval qu'elles resémantisent et qui vient se placer en altérité avec l'imaginaire médiéval traditionnel. L'analyse de l'histoire de la production de ces séries, ainsi que la prise en compte des codes télévisuels, permettent d'illustrer la spécificité du médiévalisme boréal qui se construit par l'interaction des visions exogènes, endogènes et académiques et se place dans une continuité discursive d'un imaginaire sur le Nord médiéval.

boréalisme, médiévalisme, mythologie nordique, séries télévisées, télévision, Vikings.

\section{Boreal Medievalism and TV Series}

Drawing on the concept of boreal medievalism, this article examines how the medieval North is used in TV series produced outside Nordic countries. These series testify to the heterogeneity and plasticity of the imaginary of the medieval North, which they resemantize and position as "the other" alongside the traditional medieval imaginary. Analyzing the production history of these TV series and considering televisual codes helps to illustrate the distinctive character of boreal medievalism, which is created through the interaction of exogenous, endogenous, and academic visions, and is part of a discursive continuity of an imaginary of the medieval North.

borealism, medievalism, Norse mythology, television, TV series, Vikings. 
